\title{
Apical Leakage Evaluation of Two Different Coated Carrier Systems for Root Canal Obturation Using a Dye Penetration Evaluation Method
}

\author{
Andrea Polesel ${ }^{1}$, Francesco Favaro ${ }^{2}$, Marianna G Arakelyan ${ }^{3}$, Luca Solimei ${ }^{4}$, Andrea Amaroli $^{5}$, Antonio Signore ${ }^{6}$
}

\begin{abstract}
Aim:The carrier-based obturation is a reliable technique for sealing the endodontic space. The GuttaCore with a pink internal core of cross-linked gutta-percha, named GuttaCore Pink, has been recently introduced into the market. The aim of this in vitro study was to compare the quality of the apical seal of two-carrier-based system, Soft Core and the GuttaCore Pink, through the measurement of apical dye leakage.

Materials and methods: Eighty-six extracted human teeth with single canals were used. Samples were shaped using ProTaper Universal rotary files up to a \#30 apical size and randomly divided into four groups. Group SC ( $n=40)$ was obturated with \#30 Soft Core Obturators; Group GCP $(n=40)$ was obturated with \#30 GuttaCore Pink obturators; Group CT+ $(n=3)$ and CT- $(n=3)$ left nonobturated used as positive and negative controls, respectively. The samples underwent a process of passive/active dye penetration and after a clearing procedure. The extent of the dye was measured under stereomicroscope.

Results: The Mann-Whitney U test showed a statistically significant difference $(p<0.05)$ between Soft Core and GuttaCore Pink in terms of apical dye leakage, both considering the mean and the maximum infiltration value with a greater infiltration rate for Soft Core.

Conclusion: In vitro GuttaCore Pink showed less apical dye leakage than Soft Core.

Clinical significance: The apical leakage of carrier-based obturation materials, observed in both GuttaCore Pink and Soft Core, may be considered material-dependent.

Keywords: Apical dye leakage, Apical seal, Carrier-based obturation, GuttaCore Pink, Soft core.

The Journal of Contemporary Dental Practice (2021): 10.5005/jp-journals-10024-3170
\end{abstract}

\section{INTRODUCTION}

Obturation of the endodontic space has always been a hot topic in the endodontic literature as pointed out by scientific community. ${ }^{1}$ Many techniques have been proposed, with the common goal of tridimensional filling the endodontic space, as well as to permanently seal all the exit portal of the root canal system. ${ }^{2-6}$ Among all the existing obturation methods, the carrier-based one represents a technique based on the use of warm gutta-percha that has been demonstrated to be clinically highly reliable, very easy to carry out and particularly appropriate for the obturation of long curved canals. 7,8

Resulting from this the main endodontic product manufacturer has patented their own line of obturators, each one with their own advantages.

Soft Core (Sybron Endo, Orange, California, United States) obturator is characterized by a low mass of carrier compared to the classic Thermafil obturator (Dentsply Maillefer, Ballaigues, Switzerland) and has the possibility to modify, within certain limits, the handle length and angle to facilitate the positioning in root canals with difficult access.

GuttaCore obturators (Dentsply Maillefer, Ballaigues, Switzerland) are characterized by a core made entirely of crosslinked gutta-percha which is claimed to facilitate its removal in case of retreatments or in case of postspace preparation.

Beasley et al. ${ }^{9}$ first highlighted that GuttaCore could be removed in less time from moderately curved canals using ProTaper
${ }^{1,2,4}$ Department of Surgical and Diagnostic Sciences (DISC), University of Genova, Genova, Italy

${ }^{3}$ Therapeutic Dentistry Department, Institute of Dentistry, First Moscow State Medical University (Sechenov University), Moscow, Russian Federation

${ }^{5}$ Department of Surgical and Diagnostic Sciences (DISC), University of Genova, Genova, Italy; Department of Orthopaedic Dentistry, Institute of Dentistry, First Moscow State Medical University (Sechenov University), Moscow, Russian Federation

${ }^{6}$ Department of Surgical and Diagnostic Sciences (DISC), University of Genova, Genova, Italy; Therapeutic Dentistry Department, Institute of Dentistry, First Moscow State Medical University (Sechenov University), Moscow, Russian Federation

Corresponding Author: Antonio Signore, Department of Surgical and Diagnostic Sciences (DISC), University of Genova, Genova, Italy; Therapeutic Dentistry Department, Institute of Dentistry, First Moscow State Medical University (Sechenov University), Moscow, Russian Federation, Phone: +39063721645, e-mail: dr.signore@ icloud.com

How to cite this article: Polesel A, Favaro F, Arakelyan MG, et al. Apical Leakage Evaluation of Two Different Coated Carrier Systems for Root Canal Obturation Using a Dye Penetration Evaluation Method. J Contemp Dent Pract 2021;22(9):979-984.

Source of support: Nil

Conflict of interest: None

(0) The Author(s). 2021 Open Access This article is distributed under the terms of the Creative Commons Attribution 4.0 International License (https://creativecommons. org/licenses/by-nc/4.0/), which permits unrestricted use, distribution, and non-commercial reproduction in any medium, provided you give appropriate credit to the original author(s) and the source, provide a link to the Creative Commons license, and indicate if changes were made. The Creative Commons Public Domain Dedication waiver (http://creativecommons.org/publicdomain/zero/1.0/) applies to the data made available in this article, unless otherwise stated. 
files than either Warm Vertically compacted gutta-percha or Thermafil Plus materials.

Nevares et al. ${ }^{10}$ showed in a sample of 45 maxillary central incisors that replacing a plastic core with cross-linked gutta-percha allows easier removal of the carrier from the root canal.

In addition, Endal et al. ${ }^{11}$ pointed out that GuttaCore can be removed in less time from root-end cavities than a root filling performed with a cold lateral condensation technique, and Rödig et al. ${ }^{12}$ demonstrated that regaining a working length was significantly quicker with GuttaCore when compared with Thermafil and vertically compacted gutta-percha.

A new GuttaCore with a Pink internal core of cross-linked guttapercha, named GuttaCore Pink (Dentsply Maillefer, Ballaigues, Switzerland), has been recently introduced on the market to replace the previous generation of GuttaCore, which showed risks of carrier fractures during their introduction within the canal. More recently, we showed in a previous investigation that the GuttaCore Pink can be removed from the root canal system in a shorter time when compared to the Thermafil. ${ }^{13}$

In order to evaluate the quality of the root canal filling the most commonly used method reported in the literature is the apical dye leakage test; $;^{14}$ in this study, different methods of filling ${ }^{15-19}$ are compared but rarely two-carrier-based methods. ${ }^{20,21}$ The results, expressed by the use of a score ${ }^{15,17,20,21}$ or by the use of mean and standard deviation ${ }^{16,18,19,22}$ present some sort of diskordance. In the literature, currently, there are only few studies on GuttaCore ${ }^{10-12}$ and on Soft Core ${ }^{16,20-25}$ and even less on GuttaCore Pink. ${ }^{13}$

Therefore, the aim of this research is the in vitro comparison of Soft Core and GuttaCore Pink in terms of an apical dye leakage. The null hypothesis was that there are no differences between GuttaCore Pink obturators and Soft Core obturators in terms of apical dye leakage.

\section{Materials and Methods}

This in vitro study was performed in accordance with the national low, the ethics standards of the institutional research committee and with the 1964 Helsinki declaration and its later amendments or comparable ethical standards.

\section{Sample Selection}

Eighty-six fresh extracted human teeth with single canals were selected for this investigation. The teeth were extracted for periodontal reasons. Written informed consent prior to extraction was obtained from all the patients.

The teeth recruited in this study were not previously endodontically treated, without root caries, internal and/or external root resorption and had no cracks or calcification.

Samples were horizontally sectioned at the cement-enamel junction using diamond disk mounted on straight hand piece. A\#10 Senseus FlexoFile (Dentsply Maillefer, Ballaigues, Switzerland) was used to verify the presence of only one canal, the apical patency, the absence of calcification and/or root perforations. All samples were preoperatively radiographed using digital radiographs phosphor. Samples were stored in sealed glass containers, numbered and dipped in $0.9 \%$ saline up to their use.

\section{Shaping and Cleaning Protocol}

Three endodontic motors (X-Smart, Dentsply Maillefer, Ballaigues, Switzerland) were used. The working length was determined by inserting a \#10 Senseus FlexoFile inside the canal, under visual inspection of the tip of the file approximating the major foramen, with the use of a magnification system (EyeMag Pro S, Carl Zeiss Group, Jena, Germany).

A glide path was established at first by hand instrument using a \#10 Senseus FlexoFile (Dentsply Maillefer, Ballaigues, Switzerland), then by Path File No. 1, 2, 3 rotary instruments (Dentsply Maillefer, Ballaigues, Switzerland) at $300 \mathrm{rpm}$.

For the shaping of the canal a nickel-titanium rotary technique at $300 \mathrm{rpm}$ (ProTaper Universal, Dentsply Maillefer, Ballaigues, Switzerland) was used, until a \#30 apical diameter was obtained, in order to standardize the procedure of shaping.

Glyde File Prep (Dentsply Maillefer, Ballaigues, Switzerland) was used as a lubricant before the utilization of each instrument. After each instrument a 10 seconds $2 \mathrm{~mL}$ rinse of $5 \%$ sodium hypochlorite was performed with a 27 Gauge needle, and the apical patency was verified by a \#10 a Senseus FlexoFile.

The samples underwent the following series of final rinses: $2 \mathrm{~mL}$ of $17 \%$ EDTA with a 27 Gauge needle, activating the solution with a Senseus FlexoFile \#15 passively carried $1 \mathrm{~mm}$ from the working length for a total of 1 minute, $2 \mathrm{~mL}$ of $5 \%$ sodium hypochlorite activating the solution as done with the EDTA, $2 \mathrm{~mL}$ of $0.9 \%$ saline and last rinse with $2 \mathrm{~mL}$ of alcohol.

At the end of the shaping and cleaning procedure the samples were stored in glass containers filled with $0.9 \%$ saline solution.

\section{Obturation Protocol}

Samples were randomly assigned to four groups: SC (Soft Core) $=40$ samples, GCP (GuttaCore Pink) $=40$ samples, CT+ (positive controls) $=3$ samples, CT- (negative controls) $=3$ samples.

The samples were obturated as follows: Soft Core Obturators 30.04 for the SC group and GuttaCore Pink Obturators 30.06 for the GCP group. The CT+ and CT- were shaped, but not obturated. The canals were completely dried with ProTaper Universal F3 sterile paper points (Dentsply Maillefer, Ballaigues, Switzerland).

\section{SC Group}

It was established that a \#30 Soft Core Verifier could passively arrive without interference at $0.5 \mathrm{~mm}$ from the working length as indicated by the manufacturer's instructions.

The obturators were disinfected for 60 seconds in $5 \%$ sodium hypochlorite, dried with a gauze soaked in $70 \%$ alcohol; contextually the sealer, TopSeal (Dentsply Maillefer, Ballaigues, Switzerland), was prepared and a small quantity was applied at the entrance of the canal with a sterile paper point.

The obturators were heated using the Soft Core DT Oven (Sybron Endo, Orange, California, United States) set at the heating temperature of "high" and slowly inserted, with gentle pressure, until they reached the established working length and keeping them in place for a few seconds, without cutting the handle.

\section{GCP Group}

It was established that a \#30 GuttaCore Verifier could passively arrive without interference at the working length as indicated by the manufacturer's instructions.

Disinfection and drying of the obturators and preparation of the root canal sealer were performed as described for the SC Group. The obturators were heated using the Thermaprep 2 Oven (Dentsply Maillefer, Ballaigues, Switzerland) set at the lowest heating temperature and slowly inserted, with gentle pressure, until 
they reached the established working length and keeping them in place for a few seconds, without cutting the handle.

Samples, at the end of the obturation, were stored in glass containers and incubated at $100 \%$ humidity at $37^{\circ} \mathrm{C}$ for 24 hours, in order to allow the setting of the sealer. After incubation postoperative radiographs were carried out.

\section{Dye Infiltration Protocol}

Samples of SC, GCP, and CT+ groups were isolated with two layers of nail varnish: yellow for SC samples, red for GCP samples, and white for CT+ samples, except for the last apical 2-3 mm; on the contrary, the CT- samples were fully covered with two layers of green nail varnish in order to prevent any dye penetration.

Once the varnish was dried, the specimens underwent a dye infiltration procedure as follows: during a first phase of passive infiltration, the samples were immersed in a $250 \mathrm{~mL}$ conical flask together with $100 \mathrm{~mL}$ of dye (Draw Ink, a 17, no. 201665 Pelikan, Hannover, Germany); the flask was equipped with a valve, connected, via a tube, to a pump (Wigam mv3, Wigam, Arezzo, Italy) by which the vacuum was created for 1 hour, to avoid that residual air bubble in the canal could prevent the penetration of the dye. Subsequently, the samples underwent a phase of active infiltration: they were inserted in $2 \mathrm{~mL}$ vials, filled with $1 \mathrm{~mL}$ of dye, and finally centrifuged at $1000 \mathrm{~g}$ for 5 minutes. During this stage, attention was paid so that the samples were placed with the apex pointing upwards, to ensure the penetration of dye inside the sample in case of voids.

\section{Clearing and Image Analysis}

After the infiltration procedure, the samples were rinsed with distilled water for 1 hour and the nail varnish was removed by the use of an ultrasonic scaler, curettes, and acetone.

In order to measure the extent of the infiltrate, a clearing technique as proposed by Venturi et al. ${ }^{26}$ was used.

Cleared samples were then observed with a stereomicroscope at $12 \times$ magnification (Wild m5a, Wild Heerbrugg, Switzerland) connected, via a video camera, to an image analyzer (Wild q500lw, Wild Heerbrugg, Switzerland). Sample size calculation was performed using the same method. Images on each side of the sample (buccal, lingual/palatal, mesial, distal) were acquired and the values of apical dye leakage expressed in $\mathrm{mm}$ were measured. The measurements were approximated to a 100th of a millimeter. All images were stored in JPEG format on a computer.

\section{Statistical Analysis}

Because of the asymmetry of the data distribution, descriptive analysis for continuous variables was shown as median and interquartile range; moreover, a nonparametric approach was applied.

Mann-Whitney $U$ test was carried out to determine if the difference in the infiltration value (both mean of the four surfaces and the maximum value) between GuttaCore Pink and Soft Core was significant.

The relationship between mean of the four measures and the maximum infiltration value of each sample was investigated by partial correlation, while controlling for obturation material.

Statistical significance was set at $p<0.05$ and the analyses were carried out using SPSS v.20 (SPSS Inc., Chicago, Illinois) and MedCalc v11.3 (MedCalc Software, Ostend, Belgium).

\section{Results}

Results of the study are summarized in Figure 1 and Table 1. On the total number of the analyzed samples, one of the GCP group and five of the SC group dropped out because a coronal infiltration other than apical infiltration was found. The CT+ has shown a complete infiltration, while the CT- showed no infiltration. Both considering the mean and the maximum infiltration value of the four teeth surfaces, a statistically significant difference (respectively $p=0.030$ and $p=0.020$ ) was obtained between the two endodontic materials, with a greater infiltration rate for Soft Core compared to GuttaCore Pink.

A strong positive correlation was evidenced between the mean and the maximum infiltration values $(r=+0.924, n=74, p<0.001)$ with high levels of mean infiltration associated with increased levels of the maximum values. As a consequence of the present results, the null hypothesis was rejected.

\section{Discussion}

The aim of this in vitro study was to compare the quality of the apical seal of two carrier-based system, Soft Core and the GuttaCore Pink, through the measurement of apical dye leakage. The results showed

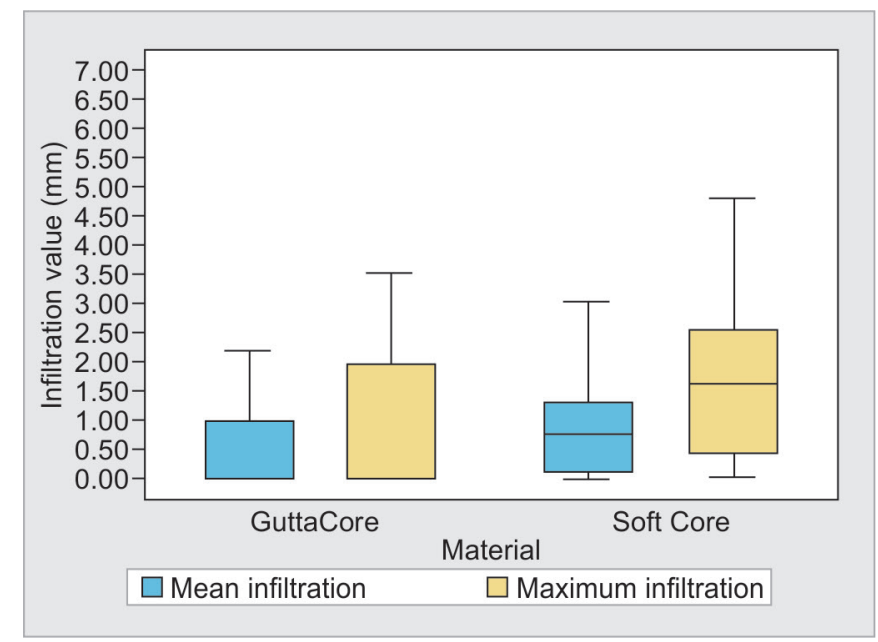

Fig. 1: Median infiltration measures for the two endodontic carrier-based obturation materials

Table 1: Differences between GuttaCore Pink and Soft Core obturation materials

\begin{tabular}{lccc}
\hline & \multicolumn{2}{c}{ Median $(I Q R)$} & \\
\cline { 2 - 3 }$n=74$ & GuttaCore Pink $(n=39)$ & Soft Core $(n=35)$ & pvalue \\
\hline Mean infiltration value $(\mathrm{mm})$ & $0.000(0.000-1.325)$ & $0.7550(0.098-1.370)$ & $0.030^{*}$ \\
Maximum infiltration value $(\mathrm{mm})$ & $0.000(0.000-2.080)$ & $1.640(0.390-2.840)$ & $0.020^{*}$ \\
\hline
\end{tabular}

"Statistically significant difference $(p<0.05)$ 
Apical Seal Ability of Two Contemporary Coated Carrier Systems

that GuttaCore Pink sustained less apical dye infiltration than Soft Core. In this respect, the results of the present study further support the outcome of previous investigations that the contemporary coated carrier systems showed comparable apical seal.

A considerable amount of research has been concerned with the sealing ability of the thermoplastic gutta-percha-coated core system Thermafil and Soft Core. ${ }^{22,24}$ An obturator based on the same idea is the GuttaCore Pink coated carrier system. Data on this latter device appear not to have been published in the scientific literature. Even if to date, there is lack of data assessing the degree of apical leakage of GuttaCore Pink; several other in vitro studies have reported good apical quality for Soft Core. Soft Core and other currently used coated carriers, such as Thermafil, showed better sealing properties than some traditional Gutta-Percha obturation techniques, including Microseal, single cone, and lateral condensation techniques. ${ }^{22,27}$ Likewise according to Gencoglu et al., ${ }^{24}$ the core (Thermafil, Quick-Fill and Soft Core) techniques and System B showed better sealing properties than Microseal and lateral condensation techniques.

Pommel and Champs $^{28}$ investigated apical sealing of singlecone, lateral condensation, vertical condensation, Thermafil, and System B obturation techniques. They found that the single cone technique produced the most apical leakage in 24 hours. They indicated that this result was attributable to the greater volume of sealer required for the single cone technique. Endodontic sealers are soluble materials and the shrinkage may result in potential leakage pathways in root canal fillings, and in fact, it has been reported that gross amounts of sealer may result in increasing amount of leakage. ${ }^{27}$ The reduced ratio of sealer to gutta-percha may improve the long-term seal provided by root canal fillings. ${ }^{22}$

Kontakiotis et al. ${ }^{29}$ investigated long-term sealing ability of different sealers and found more leakage after 2 years storage in water than before storage. In the present study, the specimens were kept in $100 \%$ humidity for 24 hours to create microleakage. Kytridou et al. ${ }^{17}$ investigated adaptation and short- and long-term seal ability of Thermafil and System B and found that both obturation techniques produced substantial filling material movement in the canal irregularities.

In literature there are conflicting results regarding the infiltration's analysis. The reasons are probably to be found in the different experimental procedures used. ${ }^{14}$

The dye used is actually a very important variable to take into consideration. In literature the most employed dyes are Black India and $2 \%$ Methylene Blue. ${ }^{30}$ However, a recent study has shown that the average size of the particles that compose these dyes is much smaller than the average size of the bacterial flora of the endodontium. Therefore, by using these dyes, it is possible to have values of infiltration that overestimate the true size of the infiltrate; ${ }^{30}$ in that study it was indicated the Draw Ink A17 Pelikan as the most suitable dye, and this is the reason why it was the dye used in our study.

Another variable is the dye infiltration method. In literature many methods have been proposed such as passive infiltration, passive after "vacuum" creation, and active infiltration. ${ }^{30,31}$ It has been hypothesized that the presence of any residual air bubbles inside the obturated canals can prevent dye infiltration. Therefore, it has been proposed to use vacuum, associated with the passive infiltration technique, in order to remove them. ${ }^{32,33}$ However, a study of Wu et al. ${ }^{30}$ has shown that it is very difficult to eliminate the air entrapped in spaces of small dimensions (such as those present in the canals), by using only a reduced pressure. Actually, another method described in the literature to remove residual air is the use of active infiltration by means of centrifugation. 14,31,34-36

Nowadays, there is no standardization of infiltration procedures. Therefore, in our study, we opted for a first phase of passive-vacuum infiltration and a subsequent phase of active infiltration by means of centrifugation. Another aspect to be taken into account during the infiltration procedure is the spatial orientation of the samples. In fact, a study by Katz et al. ${ }^{37}$ has shown that placing the samples with the apex facing upward facilitates the infiltration.

The third variable to consider is the method of analysis of the infiltrate. Many studies estimate the infiltrate in a linear manner and they consider only the highest value (in $\mathrm{mm}$ ) between the four sides of a sample (i.e., maximum infiltrate). However, this approach would not be able to estimate if the real infiltrate was a canal at threedimensional system and not a plane figure. In our work we decided to calculate a mean infiltrate value of the four sides of the sample. This approach could be more objective in assessing the behavior of the material against infiltration; for instance, sample that undergoes an infiltration of $3 \mathrm{~mm}$ on each face cannot be compared to one who suffers a penetration of only $3 \mathrm{~mm}$ on one face and different infiltration on the others.

Oliver and $\mathrm{Abbott}^{38}$ and Susini et al. ${ }^{39}$ questioned the usefulness of the methods of dye infiltration, to determine the clinical validity of a root canal obturation technique. They stated that one of the possible causes of lack of correlation between the clinical success or failure of endodontic therapy and the presence of apical infiltration is due to lack of good planning of the experimental studies. These claims are in accordance with modern concepts of Endodontics, which recognize the existence of many factors that can affect the results of treatment as stated by Ng et al. ${ }^{40}$ Although the arguments reported can be of scientific relevance, the practical and ethical need of in vitro investigations cannot be questioned; the in vitro studies should principally be used to compare two or more techniques and/or materials performance at the same laboratory conditions.

It should be pointed that the apical seal is only one among several important aspects in endodontics; indeed clinical outcome is not predictable from apical seal alone. Therefore, preclinical in vitro investigations are still needed, especially when experimental questions have to be addressed.

These considerations underline the reliability of the present study, which showed that GuttaCore Pink sustained less apical dye infiltration than Soft Core, but it is also authors' conviction that longitudinal in vivo studies with these obturation materials will be needed in order to prove their clinical effectiveness.

Some limitations should be noted. A limitation of the present study is that only two carrier-based systems were evaluated. The results of this study are therefore not directly applicable to other material formulations. Other carrier-based obturation materials with different compositions could be tested for their suitability. Furthermore, to date, there is a lack of data assessing the degree of apical leakage of one of the tested materials, and this lack of prior studies may not provide clear theoretical foundations for the research question we have investigated. In addition, the study limitations include the fact that the teeth assignment was randomized with respect to the material to be tested, but not with respect to root-canal morphology or tooth location. These limitations should be considered when interpreting the results. 


\section{Conclusion}

The results obtained in the present study justify the rejection of the null hypothesis set out previously, i.e., that there are no differences between GuttaCore Pink obturators and Soft Core obturators in terms of apical dye leakage. Within the experimental limitations of this study, it is possible to conclude that in vitro GuttaCore Pink showed less apical dye leakage than Soft Core. However, even the group that showed relatively better sealing properties was not able to completely block the ink penetration. Clinical studies are required to confirm the relevance of the present results.

\section{Clinical Significance}

This study provides evidence that the apical leakage of carrier-based obturation technique may be considered material-dependent. However, even GuttaCore Pink, which showed better sealing properties, was not able to completely prevent apical leakage.

\section{Acknowledgments}

Authors wish to thank all manufacturers for the generous donation of materials. We would also like to express our profound gratitude to Prof Edon Melloni and Prof Armanda Barbangelo from Università degli Studi di Genova, Genova, Italy, for their valuable support.

\section{References}

1. JOE Editorial Board. Obturation of the root canal system: an online study guide. J Endod 2008;34(5):e37-e43. DOI: 10.1016/j. joen.2007.06.002.

2. Schilder H. Filling root canals in three dimensions. Dent Clin N Am 1967;(Nov):723-744. PMID: 5262492

3. Bramante CM, Berbert A, Piccino AC. A technique of lateral condensation for filling root canals with gutta-percha points. Estomatol Cult 1972;6(1):70-72. PMID: 4516997.

4. Kerekes K, Rowe AH. Thermo-mechanical compaction of gutta-percha root filling. Int Endod J 1982;15(1):27-35. DOI: 10.1111/j.1365-2591.1982. tb01334.x.

5. Korzen BH. Endodontic obturation using the MicroSeal technique. Oral Health 1997;87(10):67-70. PMID: 9462131.

6. Buchanan LS. Continuous wave of condensation technique. Endod Prac 1998;1(4):7-10, 13-16, 18 passim. PMID: 10220310.

7. Johnson WB. A new gutta-percha technique. J Endod 1978;4(6):184188. DOI: 10.1016/S0099-2399(78)80173-3.

8. Leung SF, Gulabivala K. An in-vitro evaluation of the influence of canal curvature on the sealing ability of Thermafil. Int Endod J 1994;27(4):190-196. DOI: 10.1111/j.1365-2591.1994.tb00253.x.

9. Beasley $\mathrm{RT}$, Williamson $\mathrm{AE}$, Justman $\mathrm{BC}$, et al. Time required to remove guttacore, thermafil plus, and thermoplasticized gutta-percha from moderately curved root canals with protaper files. J Endod 2013;39(1):125-128. DOI: 10.1016/j.joen.2012.10.014.

10. Nevares G, de Albuquerque DS, Bueno CE, et al. Is guttacore more easily removed from the root canal than thermafil? An ex-vivo study. J Canad Dent Ass 2015;81:f22. PMID: 26679336.

11. Endal U, Shen Y, Ma J, et al. Evaluation of quality and preparation time of retrograde cavities in root canals filled with guttacore and cold lateral condensation technique. J Endod 2018;44(4):639-642. DOI: 10.1016/j.joen.2017.11.023.

12. Rödig T, Wagner J, Wiegand A, et al. Efficacy of the ProTaper retreatment system in removing Thermafil, GuttaCore or vertically compacted gutta-percha from curved root canals assessed by micro-CT. Int Endod J 2018;51(7):808-815. DOI: 10.1111/iej.12893.

13. Fracchia DE, Amaroli A, De Angelis N, et al. GuttaCore Pink, Thermafil and Warm Vertically compacted gutta-percha retreatment: time required and quantitative evaluation by using ProTaper files. Dent Mat J 2020;39(2):229-235. DOI: 10.4012/dmj.2019-008.

14. Wu MK, Wesselink PR. Endodontic leakage studies reconsidered. Part I. Methodology, application and relevance. Int Endod J 1993;26(1): 37-43. DOI: 10.1111/j.1365-2591.1993.tb00540.x.

15. Gutmann JL, Saunders WP, Saunders EM, et al. An assessment of the plastic Thermafil obturation technique. Part 2. Material adaptation and sealability. Int Endod J 1993;26(3):179-183. DOI: 10.1111/j.13652591.1993.tb00790.x.

16. De Moor RJ, Martens LC. Apical microleakage after lateral condensation, hybrid gutta-percha condensation and Soft Core obturation: an in vitro evaluation. Endod Dent Traumatol 1999;15(5):239-243. DOI: 10.1111/j.1600-9657.1999.tb00780.x.

17. Kytridou V, Gutmann JL, Nunn MH. Adaptation and sealability of two contemporary obturation techniques in the absence of the dentinal smear layer. Int Endod J 1999;32(6):464-474. DOI: 10.1046/j.13652591.1999.00248.x.

18. Abarca AM, Bustos A, Navia M. A comparison of apical sealing and extrusion between Thermafil and lateral condensation techniques. J Endod 2001;27(11):670-672. DOI: 10.1097/00004770-20011100000004.

19. Inan U, Aydemir H, Tasdemir T. Leakage evaluation of three different root canal obturation techniques using electrochemical evaluation and dye penetration evaluation methods. Aust Endod J 2007;33(1):18 22. DOI: $10.1111 /$ j.1747-4477.2007.00050.x.

20. De Moor RJ, De Boever JG. The sealing ability of an epoxy resin root canal sealer used with five gutta-percha obturation techniques. Endod Dent Traumatol 2000;16(6):291-297. DOI: 10.1034/j.16009657.2000.016006291.x.

21. De Moor RJ, Hommez GMG. The long-term sealing ability of an epoxy resin root canal sealer used with five gutta-percha obturation techniques. Int Endod J 2001;35(5):275-282. DOI: 10.1046/j.13652591.2002.00481.x.

22. Gencoglu N, Garip Y, Bas M, et al. Comparison of different guttapercha root filling techniques: Thermafil, Quick-fill, System B, and lateral condensation. Oral Surg Oral Med Oral Pathol Oral Radiol Endod 2002;93(3):333-336. DOI: 10.1067/moe.2002.120253.

23. Boussetta F, Bal S, Romeas A, et al. In vitro evaluation of apical microleakage following canal filling with a coated carrier system compared with lateral and thermomechanical Gutta-Percha condensation techniques. Int Endod J 2003;36(5):367-371. DOI: 10.1046/j.1365-2591.2003.00665.x.

24. Gencoglu N, Orucoglu H, Helvacioglu D. Apical leakage of different gutta-percha techniques: Thermafil, Js Quick-Fill, Soft Core, Microseal, System B and lateral condensation with a computerized fluid filtration meter. Eur J Dent 2007;1(2):97-103. PMCID: PMC2609951.

25. Ozer SY, Aktener BO. Outcome of root canal treatment using Soft Core and cold lateral compaction filling techniques: a randomized clinical trial. J Contemp Dent Pract 2009;10(1):74-81. PMID: 19142259.

26. Venturi M, Prati C, Capelli G, et al. A preliminary analysis of the morphology of lateral canals after root canal filling using a toothclearing technique. Int Endod J 2003;36(1):54-63. DOI: 10.1046/ j.0143-2885.2003.00613.x.

27. Wu MK, van der Sluis LW, Wesselink PR. A preliminary study of the percentage of gutta-percha-filled area in the apical canal filled with vertically compacted warm gutta-percha. Int Endod J 2002;35(6):527-535. DOI: 10.1046/j.1365-2591.2002.00522.x.

28. Pommel L, Camps J. In vitro apical leakage of system B compared with other filling techniques. J Endod 2001;27(7):449-451. DOI: 10.1097/00004770-200107000-00003.

29. Kontakiotis EG, Wu MK, Wesselink PR. Effect of sealer thickness on long-term sealing ability: a 2-year follow-up study. Int Endod J 1997;30(5):307-312. DOI: 10.1046/j.1365-2 591.1997.00087.x.

30. Wu MK, De Gee AJ, Wesselink PR. Fluid transport and dye penetration along root canal fillings. Int Endod J 1994;27(5):233-238. DOI: 10.1111/j.1365-2591.1994.tb00261.x. 
31. Pathomvanich S, Edmunds DH. Variation in the microleakage produced by four different techniques in root fillings in a simulated root canal model. Int Endod J 1996;29(3):156-162. DOI: 10.1111/j.13652591.1996.tb01362.x.

32. Spangberg LS, Acierno TG, Yongbum Cha B. Influence of entrapped air on the accuracy of leakage studies using dye penetration methods. J Endod 1989;15(11):548-555. DOI: 10.1016/s0099-2399(89)80199-2.

33. Wimonchit S, Timpawat S, Vongsavan N. A comparison of techniques for assessment of coronal dye leakage. J Endod 2002;28(1):1-4. DOI: 10.1097/00004770-200201000-00001.

34. Oliver CM, Abbott PV. Entrapped air and its effects on dye penetration of voids. Endod Dent Traumatol 1991;7(3):135-138. DOI: 10.1111/j.16009657.1991.tb00198.x.

35. Limkangwalmongkol S, Abbott PV, Sandler AB. Apical dye penetration with four root canal sealers and gutta-percha using longitudinal sectioning. J Endod 1992;18(11):535-539. DOI: 10.1016/S00992399(06)81209-4.
36. Karagoz-Kucukay I, Kucukay S, Bayirli G. Factors affecting apical leakage assessment. J Endod 1993;19(7):362-365. DOI: 10.1016/S00992399(06)81364-6.

37. Katz A, Rosenwasser $R$, Tamse A. Root positioning and leakage to dye in extracted teeth using reduced pressure. Int Endod J 1998;31(1): 63-66. PMID: 9823131.

38. Oliver CM, Abbott PV. Correlation between clinical success and apical dye penetration. Int Endod J 2001;34(8):637-644. DOI: 10.1046/j.13652591.2001.00442.x.

39. Susini G, Pommel L, About I, et al. Lack of correlation between ex vivo apical dye penetration and presence of apical radiolucencies. Oral Surg Oral Med Oral Pathol Oral Radiol Endod 2006;102(3):e19-e23. DOI: 10.1016/j.tripleo.2006.03.015.

40. Ng YL, Mann V, Gulabivala K. A prospective study of the factors affecting outcomes of nonsurgical root canal treatment: part 1 periapical health. Int Endod J 2011;44(7):583-609. DOI: 10.1111/ j.1365-2591.2011.01872.x. 